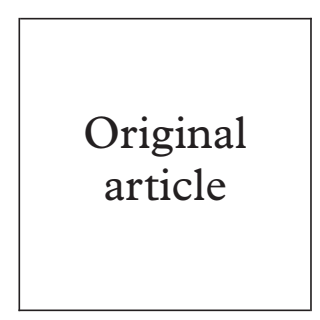

\title{
First episodes of genital herpes in a Swedish STD population: a study of epidemiology and transmission by the use of herpes simplex virus (HSV) typing and specific serology
}

\author{
G-B Löwhagen, P Tunbäck, K Andersson, T Bergström, G Johannisson
}

Objectives: To determine the proportion of herpes simplex virus type 1 (HSV-1) and HSV type 2 (HSV-2) in first episodes of genital herpes. To evaluate the use of HSV specific serology for classifying first episodes of genital herpes and for defining HSV serostatus in the patients' sexual partners.

Methods: 108 consecutive patients with first episodes of genital herpes seen at three STD clinics in Sweden from 1995 to 1999 were included in the study. HSV culture and typing were performed and serum was tested for antibodies against a type common HSV antigen and a type specific HSV-2 antigen, glycoprotein G2 (gG2). A structured interview including questions about sexual behaviour and sexual partners was taken. "Steady" partners were offered a blood test for HSV serology and counselling.

Results: Of 108 patients, 11 had a negative HSV culture. Of the 97 who were HSV culture positive, $44 \%$ (43/97) were typed as HSV-1 and 56\% (54/97) as HSV-2. For 86 of these 97 patients, HSV serology from the initial visit was available. Of 52 primary infections, thus initially seronegative, $64 \%$ were HSV-1 infections and of 19 female primary infections 16 (84\%) were HSV-1. In $17 \%$ the first episode of genital herpes corresponded to the first clinical recurrence of an infection acquired earlier in life. There was a significant correlation between having orogenital sex and being infected with HSV-1 and also a history of labial herpes in the partner. Only $20 \%$ of partners of patients with an HSV-2 infection had a history of genital herpes.

Conclusions: Almost half of first episodes of genital herpes are caused by HSV-1. In young women with a primary genital infection, HSV-1 is much more frequent than HSV-2. Besides HSV typing, we found specific HSV serology of value for classifying first episodes and for diagnosing a subclinical HSV-2 infection in partners. Anamnestic data supported the suggestion that the orogenital route of transmission was common in genital HSV-1 infections.

(Sex Transm Inf 2000;76:179-182)

Keywords: genital herpes; herpes simplex virus

Departments of

Dermatology and

Venereology and

Clinical Virology

Sahlgrenska University

Hospital, Göteborg

and the STD Clinic,

Borås Central

Hospital, Sweden

G-B Löwhagen

P Tunbäck

K Andersson

T Bergström

G Johannisson

Correspondence to: Dr Gun-Britt Löwhagen, Sahlgrenska University

Hospital, 41345 Göteborg,

Sweden

Gun-Britt.Lowhagen@

sahlgrenska.se

Accepted for publication 25 February 2000

\section{Introduction}

In addition to the common manifestation of herpes simplex virus (HSV) type 1 as orolabial herpes, HSV-1 is being increasingly recognised as a cause of genital infection. ${ }^{1-3}$ The vast majority of HSV infections, both orolabial and genital, are asymptomatic. ${ }^{45}$ Serological tests that discriminate between HSV-1 and HSV-2 infections have been developed and subclinical carriers of HSV can be identified. ${ }^{67}$ Genital herpes is a disease with high psychosexual morbidity and a great concern to the patient is from whom and when they contracted their infection. ${ }^{8}$ The primary aim of the present study was to evaluate the prevalence of HSV types 1 and 2 in first episodes of genital herpes in a Swedish STD population. Further, we wanted to investigate the value of type specific HSV serology for clarifying routes of transmission in order to be able to give better counselling to infected patients and their partners.

\section{Patients and methods}

Consecutive patients with a clinical picture and history compatible with a first episode of genital herpes seen by the authors at the STD clinics of Sahlgrenska University Hospital, Göte- borg and Borås Central Hospital, Borås between November 1995 and March 1999 were recruited to the study. In Göteborg about 4000 new attenders are seen annually at the STD clinics and in Borås about 1000. In both areas younger patients ( $<25$ years old) often attend special youth clinics and many women attend a gynaecological clinic, which explains the dominance of men. The age and sex of the study population does not differ from the overall STD population in our clinics.

Altogether 108 heterosexual individuals, 67 men and 41 women, were included. The age of the men ranged from 19 to 54 years mean 31, compared with $17-40$ years, mean 26 , for the women $(p<0.001)$. Specimens for HSV culture were collected and blood samples for HSV serology were drawn. A structured interview including questions about sexual behaviour and sexual partners was taken. A steady partner was defined as a partner with whom the patient had an ongoing sexual relationship for at least 2 months. History of labial or genital herpes in steady partners was asked for and they were offered examination, counselling, and a blood test for HSV serology. 
ISOLATION AND TYPING OF VIRUS

Material from blisters or eroded lesions was collected with a cotton tipped (ENT) swab and at the laboratory directly inoculated on cultures of green monkey kidney cells. Cytopathic effects were registered daily and after passage typing of isolated virus was performed by means of enzyme linked immunosorbent assay (ELISA) using monoclonal antibodies type specifically reacting with HSV type 2 glycoprotein $\mathrm{G}$ (gG-2) or with HSV type 1 glycoprotein C. ${ }^{9}$

SEROLOGY

Antibodies reacting with type common and type 2 specific (gG-2) antigen were assayed by ELISA methods. ${ }^{10}$

Based on results of culture and acute phase serology, first episodes of genital herpes were classified as primary, non-primary, or recurrent infection as follows ${ }^{7}{ }^{11}$ :

\begin{tabular}{|c|c|c|c|}
\hline & $\begin{array}{l}H S V \\
\text { typing }\end{array}$ & $\begin{array}{l}\text { Antibodies } \\
\text { against HSV } \\
\text { common ag }\end{array}$ & $H S V-2$ \\
\hline Primary HSV-2 & HSV-2 & neg & neg \\
\hline Non-primary HSV-2 & HSV-2 & pos & neg \\
\hline First clinical recurrence HSV-2 & HSV-2 & pos & pos \\
\hline Primary HSV-1 & HSV-1 & neg & neg \\
\hline Non-primary HSV-1 & HSV-1 & pos & neg/pos \\
\hline First clinical recurrence HSV-1 & HSV-1 & pos & neg/pos \\
\hline
\end{tabular}

Concerning HSV-1 infections, serology could not discriminate between a non-primary and a recurrent infection.

STATISTICAL METHODS

Fisher's exact test, the $t$ test and a logistic regression analysis were used and $\mathrm{p}<0.05$ was considered significant.

The study was approved by the ethics committee of the medical faculty of Göteborg University.

\section{Results}

HSV CULTURE/TYPING

Of the 108 patients, 97 had a positive culture for HSV. In 44\% (43/97) the isolate was typed as HSV-1 and in 57\% (54/97) as HSV-2 (table 1). HSV-1 was isolated from 53\% (20/38) of genital lesions in women compared with $39 \%$ $(23 / 59)$ in men (NS). The mean age for patients with HSV-1 was 27 years (range 17-51), compared with 31 years (range 18-53) for those with an HSV-2 infection $(p=0.016)$. To exclude covariation between sex and age, type was tested as function of age and sex in a logistic regression analysis and a significant correlation $(p=0.04)$ between type and age was still demonstrated but not between HSV type and sex.

Table 1 Sex and age of 97 patients with first episode genital herpes, 43 HSV-1 and 54 HSV-2 infections

\begin{tabular}{llllll}
\hline & \multicolumn{2}{l}{ Male patients } & & \multicolumn{2}{l}{ Female patients } \\
\cline { 2 - 3 } \cline { 6 - 6 } \cline { 6 - 6 } & $H S V-1$ & $H S V-2$ & & $H S V-1$ & $H S V-2$ \\
\hline $\begin{array}{l}\text { Number } \\
\begin{array}{l}\text { Age (years) } \\
\text { mean } \\
\text { range }\end{array}\end{array}$ & 23 & 36 & & 20 & 18 \\
\hline
\end{tabular}

Table 2 Age and sex in 52 cases of primary HSV infection

\begin{tabular}{llllll}
\hline & \multicolumn{2}{l}{ Male patients } & & \multicolumn{2}{l}{ Female patients } \\
\cline { 2 - 3 } \cline { 6 - 6 } \cline { 5 - 6 } & $H S V-1$ & HSV-2 & & HSV-1 & HSV-2 \\
\hline $\begin{array}{l}\text { Number } \\
\begin{array}{c}\text { Age (years) } \\
\text { mean }\end{array}\end{array}$ & 17 & 16 & & 16 & 3 \\
range & 29.5 & 29.4 & & 23.9 & 25.3 \\
\hline
\end{tabular}

CLASSIFICATION OF FIRST EPISODES OF GENITAL HERPES

Of 97 patients with positive culture, serology was available from the initial visit in 86 . First episodes in these patients were classified as primary in $52(61 \%)$, non-primary HSV-2 in 11 (13\%), recurrent $\mathrm{HSV}-2$ in 15 (17\%), and non-primary or recurrent HSV-1 in eight $(9 \%)$.

Recurrent infection in this context corresponds to the first episode of clinical symptoms of a previous HSV infection. Of 52 primary infections, $64 \%$ (33/52) were HSV-1 infections, and of female primary infections $84 \%$ (16/19) were HSV-1 (table 2). Women with primary infection were significantly younger than men $(p=0.02)$. When type was tested as a function of age and sex in a logistic regression analysis there was a significant correlation between type and sex $(\mathrm{p}=0.04)$ but not between type and age.

\section{PARTNERS}

Of $40 \mathrm{HSV}-1$ index cases (for three patients data were missing), 31 (78\%) answered that they had a steady sexual partner and denied casual sexual contacts during the past 2 months and of $54 \mathrm{HSV}-2$ index cases $38(70 \%)$ had a stable sexual relationship. About half of these partners were seen at the clinic, mostly within 2 weeks after the index patient's visit, and 29 agreed to have a blood test taken for serological analysis.

The prevalences of labial and genital herpes in partners of HSV-1 and HSV-2 index cases are shown in table 3. Answers to these questions were not available for all steady partners. History of labial herpes was significantly $(p=0.02)$ more frequent in partners of HSV-1 infected patients.

Of $26 \mathrm{HSV}-1$ infected patients, all but one who answered the question about orogenital sex reported having practised orogenital sex in connection with onset of the infection, compared with eight $(57 \%)$ of 14 in the HSV-2 group $(p=0.004)$.

HSV serology was available for 15 partners to index patients with HSV-1 infection and for 14 partners to HSV-2 infected patients, all but one without a history of genital herpes (table 4). Of partners to HSV-1 infected patients, $7 \%$ (1/15) had antibodies to HSV-2, compared with $71 \%(10 / 14)$ of partners to HSV-2 infected patients $(p=0.0005)$. Infections in the index patients to the four seronegative partners were classified as one recurrence, one nonprimary HSV-2, and two primary infections. Thus, in three cases, two primary and one non-primary HSV-2 infection, the partners were seronegative for HSV-2 in spite of being the only plausible source of infection (the index 
Table 3 History of labial and genital herpes in 69 consorts to index patients with first episode genital herpes

\begin{tabular}{lll}
\hline & Labial herpes & Genital herpes \\
\hline Partners to index patients with HSV-1 $(\mathrm{n}=31)$ & $15 / 28(50 \%)$ & $2 / 30(11 \%)$ \\
Partners to index patients with HSV-2 $(\mathrm{n}=38)$ & $5 / 24(21 \%)$ & $7 / 32(22 \%)$ \\
\hline
\end{tabular}

Table 4 HSV serology in 29 partners to index patients with genital herpes

\begin{tabular}{lll}
\hline & \multicolumn{2}{l}{ Number with antibodies against } \\
\cline { 2 - 3 } & HSV common antigen & gG2 antigen \\
\hline Partners to HSV-1 index cases $(\mathrm{n}=15)$ & $14 / 15$ & $1 / 15$ \\
Partners to HSV-2 index cases $(\mathrm{n}=14)$ & $11 / 14(79 \%)$ & $10 / 14(71 \%)$ \\
\hline
\end{tabular}

patients denied any other sexual partners within at least 2 months). Sera in these couples were further analysed for HSV-2 by western blotting (WB). In all three index cases, WB disclosed a weak band corresponding to gG2 while all three partners were negative in WB for gG2. The times since onset of the clinical infection in the index cases were 2, 4, and 10 days respectively.

\section{Discussion}

An increasing number of patients attending STD clinics, general practices, and gynaecological clinics with a diagnosis of genital herpes has been noted since the 1970s both in Europe and in the United States. ${ }^{1}{ }^{12} 13$

Although HSV-2 remains a predominantly genital pathogen, HSV-1 has been variably reported as causing between $4 \%$ and $60 \%$ of genital herpes episodes. ${ }^{14-18}$ In a recent prospective study of sexually active HSV-2 seronegative people the rates of new HSV-1 and HSV-2 infections were 1.6 and 5.1 cases per 100 person years, respectively. ${ }^{19}$ An increase of genital HSV-1 infections has been reported, especially from the United Kingdom. ${ }^{23}{ }^{20}$ Specific HSV serology has been available since the 1980s and contributed considerably to our understanding of the epidemiology, especially of HSV-2 infections. ${ }^{6721}$ A recent seroepidemiological study of the population from the United States demonstrated an increasing incidence of HSV-2 infections, especially in young adults. ${ }^{22}$ This is in contrast with serological data from Sweden, which indicate a decreasing incidence in young women. ${ }^{23}$

While HSV-2 antibodies with few exceptions reflect a genital infection, HSV-1 antibodies may be evoked by either an oral infection or a genital infection or both. Thus, serology alone is not well suited to studying the prevalence of genital HSV-1 infections.

It is well known that an HSV-1 genital infection gives less frequent clinical recurrences than an HSV-2 infection. ${ }^{7}$ Thus, when estimating the proportion of HSV-1 in a population of genital herpes it is of importance to know if the study group includes only primary infections, first episode genital herpes, or all cases of genital herpes diagnosed, including recurrences. By performing type specific HSV serology on sera from the initial visit, it is possible in most cases to discriminate between primary/non-primary infections and recurrences. ${ }^{11}$ Convalescent sera may also be of value but seroconversion is often late, especially if antiviral therapy is given. ${ }^{24}$

In the present study comprising 97 patients with their first episode of clinical genital herpes, $43 \%$ were caused by HSV-1 and when evaluating only "true" primary infections (seronegative at the initial visit) the prevalence of HSV-1 was $64 \%$. In $17 \%$ the infection was a recurrence - that is, the first clinical manifestation of an earlier acquired infection. The severity of the lesions was not systematically registered in this study. Although some cases of primary infections had a severe course, with fever and systemic symptoms, it was not possible in most cases to differentiate between a primary, non-primary, and recurrent HSV infection from the clinical picture. The dominance of men in our patients reflects the male/female proportion of attenders at our clinic since many women in our region consult a gynaecologist.

A simple explanation why genital HSV-1 has become more common could not be presented. One reason may be that HSV infections during childhood seem to have decreased. The lower prevalence of HSV antibodies means that more young adults are seronegative for HSV at their sexual debut and thus more susceptible. However, seroprevalence data supporting this theory are scanty ${ }^{25-27}$ and in Swedish pregnant women the prevalence of HSV-1 antibodies had not changed from 1969 to $1989 .{ }^{28}$ Changes in sexual behaviour may have importance and orogenital sex is probably more frequently practised as a consequence of "safer sex" programmes in the HIV campaigns. A third explanation could be a change in viral pathogenicity.

None of these proposed theories could explain why HSV-1 infection is more common in especially younger women than in men. It seems unlikely that more frequent practice of cunnilingus relative to fellatio could explain this disparity between the sexes.

Several seroepidemiological studies have shown a significantly higher prevalence of HSV-2 antibodies in women compared with men. ${ }^{17} 20$ A possible explanation could be the different anatomy in men and women. Besides exposing a larger area, the female genital mucosa/skin may be more susceptible to HSV, both HSV-1 and HSV-2, compared with the keratinised penile epithelium. A similar sex difference has been reported for HIV, with a higher risk of male to female than female to male transmission. ${ }^{29}$

In most cases, the patient has acquired the infection from someone who is not aware of having either labial or genital herpes. ${ }^{30}$ Asymptomatic shedding of HSV is a frequent occurrence both from oral mucosa/saliva ${ }^{4}$ and genital mucosa/skin. ${ }^{5}$ The diagnosis of genital herpes gives rise to much distress in the patient. In a stable relationship, a great concern could be from where the infection originates. In the present study, a silent HSV-2 infection could be diagnosed by serology in about $70 \%$ of the partners and in the index patient recurrent and primary infections could be discriminated. A history of orogenital sexual contact was more frequently reported in cases of 
HSV-1 genital herpes, which does not exclude the possibility of genital-genital transmission. The HSV-2 antibody test using gG2 as antigen has been thoroughly validated and the sensitivity is high in culture proved recurring genital infections. ${ }^{31}$ Western blot is referred to as the golden standard and has a higher sensitivity to detect antibodies especially in primary infection. ${ }^{32}$ It is important to be aware of the limitations of these tests as illustrated by three cases in this study. Our knowledge concerning the natural course of genital HSV infection and its evoked immune response is still limited. Do all HSV infections elicit a humoral response? How long could the interval be from inoculation of the virus until reactivation, serological response, and/or clinical infection? Prospective studies including repeated HSV serology and genital sampling for PCR of seronegative partners to patients with genital herpes may shed light on these issues, although these studies for practical and economical reasons are difficult to perform.

In our opinion, in spite of the shortcomings with available tests, HSV typing as well as HSV serology improved and facilitated counselling of the patient and the partner. An interview study to evaluate the psychological effect of serological testing, becoming aware of HSV-2 serostatus, in the partners is in progress.

This work was supported by the regional health authority of western Sweden and the LUA foundation of Sahlgrenska University Hospital.

Contributors: The study was designed by GBL and PT; TB and PT were responsible for performance and interpretation of the laboratory tests and for valuable comments in the development of the mansuscript; KA and GJ participated by collecting
patient data and by giving comments on the study design; and the final manuscript was prepared by GBL.

1 Tayal SC, Pattman RS. High prevalence of herpes simplex virus type 1 in female anogenital herpes simplex in Newcastle upon Tyne 1983-92. Int $\mathcal{F}$ STD AIDS 1994;5:359-61.

2 Ross JDC, Smith IW, Elton R. The epidemiology of herpes simplex types 1 and 2 infection of the genital tract in Edinburgh 1978-1991. Genitourin Med 1993;69:381-3.

3 Rodgers CA. High prevalence of herpes simplex virus type 1 in female anogenital herpes. Int $\mathcal{F}$ STD AIDS 1995;6:144 6 .

4 Spruance SL. Pathogenesis of herpes simplex labialis: excreSpruance SL. Pathogenesis of herpes simplex labialis: excre-
tion of virus in the oral cavity. 7 Clin Microbiol tion of virus

5 Wald A, Zeh J, Selke S, et al. Virological characteristics of subclinical and symptomatic genital herpes infections. $N$ Engl f Med 1995;21:770-4.

6 Bergström T, Trybala E. Antigenic differences between HSV-1 and HSV-2 glycoproteins and their importance of type-specific serology. Intervirology 1996;39:176-84.

7 Corey L. The current trend in genital herpes. Progress in prevention. Sex Transm Dis 1994;21(suppl):38-44.

8 Mindel A. Psychological and psychosexual implications of herpes simplex virus infections. Scand F Infect Dis (Suppl) 1996;100:27-32.

9 Liljeqvist JA, Svennerholm B, Bergstrom T. Typing of clinical herpes simplex virus type 1 and type 2 isolates with monoclonal antibodies. $\mathcal{F}$ Clin Micrbiol 1999;37:2717-8.
10 Svennerholm B, Olofsson S, Jeansson S, et al. Herpes simplex virus type-selective enzyme-linked immunosorbent assay with Helix pomatia-lectin purified antigens. $f$ Clin Microbiol 1984;19:235-9.

11 Bernstein DI, Lovett MA, Bryson YJ. Serological analysis of first-episode nonprimary genital herpes simplex virus infection. Presence of type 2 antibody in acute serum samples. Am f Med 1984;77:1055-60.

12 Kinghorn GR. Limiting the spread of genital herpes. Scand F Infect Dis (Suppl) 1996;100:20-5.

13 Becker TM, Stone KM, Cates W Jr. Epidemiology of genital herpes infections in the United States. The current situation. F Reprod Med 1986;31(Suppl):359-64.

14 Nahmias AJ, Dowdle WR, Naib ZM, et al. Genital infection with type 2 herpes virus hominis. A commonly occuring venereal disease. Brf Vener Dis 1969;45:294-8.

15 Cheong WK, Thiurumoorthy T, Doraisingham S, et al. Clinical and laboratory study of first episode genital herpes in Singapore. Int $\mathcal{F}$ STD AIDS 1990;1:195-8.

16 Lavery HA, Connolly JH, Russell JD. Incidence of herpes simplex in Northern Ireland in 1973-83 and and herpes simplex types 1 and 2 isolated in 1982-4. Genitourin Med 1986;62:24-7.

17 Löwhagen GB, Jansen E, Nordenfelt E, et al. Epidemiology of genital herpes in Sweden. Acta Derm Venereol (Stockh) 1990;70:330-4.

18 Wooley PD, Kudesia G. Incidence of herpes simplex virus type- 1 and type-2 from patients with primary (first-attack) genital herpes in Sheffield. Int F STD AIDS 1990;1:184-6.

19 Langenberg AG, Corey L, Ashley RL, et al.A prospective study of new infections with herpes simplex virus type 1 and type 2. Chiron HSV Study Group. N Engl f Med 1999; 341:1432-8

20 Christie SN, McCaughey C, McBride M, et al. Herpes simplex type 1 and genital herpes in Northern Ireland. Int $\mathcal{F}$ STD AIDS 1997;8:68-70.

21 Cowan MF, Johnson AM, Ashley R, et al. Relationship between antibodies to herpes simplex virus (HSV) and symptoms of HSV infection. F Infect Dis 1996;174:470-5.

22 Fleming DT, McQuillan GM, Johnson RE, et al. Herpes simplex virus type 2 in the United States, 1976 to 1994 . N Engl f Med 1997;337:1105-11.

23 Persson K, Månsson A, Jönsson E, et al. Decline of herpes simplex virus type 2 and Chlamydia trachomatis infections from 1970 to 1993 indicated by a similar change in antibody pattern. Scand F Infect Dis 1995;27:195-9.

24 Ashley RL, Corey L. Effect of acyclovir treatment of primary genital herpes on the antibody response to herpes implex virus. $\mathcal{F}$ Clin Invest 1984;73:681-8.

25 Brown DWG, Vys EA, Slomka M, et al. An age-stratified survey of antibody to HSV type-1 and type- 2 in the UK using monoclonal antibody blocking assays (abstract). 12th Meeting of the ISSTDR, Seville, 1997.

26 Smith IW, Peutherer JF, MacCallum FO. The incidence of herpes virus hominis antibody in the population. $f$ Hyg 1967;65:395-408.

27 Wolontis S. Infection with herpes simplex viruses types 1 and 2. N Engl f Med 1973;289:781-9.

28 Forsgren M, Skoog E, Jeanson S, et al. Prevalence of antibodies to herpes simplex virus in pregnant women in Stockholm in 1969, 1983 and 1989: implications for STD epidemiology. Int f STD AIDS 1994;5:113-6.

29 Nicolosi A, Correa Leite ML, Musicco M, et al. The efficiency of male-to-female and female-to-male sexual transmission of the human immunodeficiency virus: a study of 730 stable couples. Italian study group on HIV heterosexual transmission. Epidemiology 1994;5:570-5.

30 Mertz GJ, Benedetti J, Ashley R, et al. Risk factors for the sexual transmission of genital herpes. Ann Intern Med 1992;116:197-202.

31 Ho DW, Field PR, Sjogren-Jansson E, et al. Indirect ELISA for the detection of HSV-2 specific IgG and IgM antibodies with glycoprotein G (gG-2). F Virol Methods 1992;36: 249-64.

32 Ashley RL, Militoni J, Lee F, et al .Comparison of Western blot (immunoblot) and glycoprotein G-specific immunodot enzyme assay for detecting antibodies to herpes simplex virus types 1 and 2 in human sera. 7 Clin Microbiol 1988;26:662-7. 